\title{
Corrigendum: Generation of subnanometric platinum with high stability during transformation of a 2D zeolite into 3D
}

\author{
Lichen Liu, Urbano Díaz, Raul Arenal, Giovanni Agostini, Patricia Concepción and Avelino Corma
}

Nature Materials 16, 132-138 (2017); published online 26 September 2016; corrected after print 24 March 2017 and 25 October 2017

In the version of this Article originally published, the four instances of the window type on page 136 should have read ' 10 -MR'. On the same page, in the 'Catalytic activity' section, the accessibility of isobutene was incorrectly described, and the phrase should have read 'isobutene is mainly accessible to Pt species located at the external cups on the surface of MCM-22'. These changes do not affect the results of the Article.

\section{Addendum: Magnetically assisted slip casting of bioinspired heterogeneous composites}

Hortense Le Ferrand, Florian Bouville, Tobias P. Niebel and André R. Studart

Nature Materials 14, 1172-1179 (2015); published online 21 September 2015; corrected after print 10 November 2017

In the original version of this Article, the crack-growth resistance (R-curve) of nacre-like materials produced by magnetically assisted slip casting was quantified following published guidelines (M. E. Launey, Acta Mater. 57, 2919-2932; 2009) and the conservative recommendations of the 2013 ASTM E1820 standard (Standard Test Method for the Measurement of Fracture Toughness). Such guidelines determine the maximum crack extension below which toughness values should be valid. However, although the nacre-like specimens with polymer and metal matrices show typical rising crack-growth resistance of toughened brittle materials, the R-curves for the all-ceramic samples exhibit an upward concave appearance at high crack extensions (Fig. $3 \mathrm{f}$ in the Article). This concern was raised by Robert Ritchie (Lawrence Berkeley National Laboratory). Although the prevalent toughening mechanism in the authors' nacrelike alumina is crack deflection rather than large-scale bridging, the upward shape of the curve might originate from specimen-size effects. In contrast to what occurs for other nacre-like materials, this concave shape appears in the authors' samples at crack extensions $(\Delta a)$ much lower than the conservative limit recommended by the standard ( $\Delta a_{\max }=0.4 \mathrm{~mm}$; Fig. 3f in the Article). The challenge in this particular type of measurement is to keep the main crack under conditions that fulfil the fracture-mechanics arguments assumed in the calculations.

Following Ritchie's justified recommendation, the authors have now revisited the R-curve data for this specific set of samples to obtain an even more conservative value for the crack-growth resistance. Taking into account the maximum fracture toughness obtained in the linear regime (before the upswing), they obtain an average $K_{\mathrm{IC}}$ of $11.3 \pm 2.4 \mathrm{MPa} \mathrm{m}{ }^{1 / 2}$ for the nacre-like alumina specimens. This value is in close agreement with the average apparent fracture toughness $K_{\mathrm{IR}}^{\mathrm{a}}$ of $11.5 \pm 0.5 \mathrm{MPa} \mathrm{m}{ }^{1 / 2}$ calculated from the peak load of the force-displacement curves, which is not corrected for crack extension and thus renders a conservative value for the crack-growth resistance. On average, these conservative numbers are approximately $18 \%$ lower than what is reported in the original version of the Article $\left(14 \mathrm{MPa} \mathrm{m}^{1 / 2}\right)$. Figure $3 \mathrm{f}$ has therefore been corrected (shown here). For consistency, the following change has been made to the text. On page 1177, the text "Remarkably, the fracture toughness $\left(K_{\mathrm{IC}}\right)$ of the nacre-like alumina $\left(14 \mathrm{MPa}^{1 / 2}\right)$ increases by a factor of 3 to 4 compared with that of untextured fine-grained alumina, whereas the fracture strength is maintained at levels comparable to the strongest aluminas $(650 \mathrm{MPa})$ " has been changed to read "Remarkably, the fracture toughness $\left(K_{\mathrm{IC}}\right)$ of the nacre-like alumina (11.5 MPa $\mathrm{m}^{1 / 2}$ ) increases by a factor of three compared with that of untextured fine-grained alumina, whereas the fracture strength is maintained at levels comparable to the strongest aluminas (650 MPa)".

To evaluate whether size effects play a role on the crack-growth resistance of our nacre-like alumina, the authors prepared larger specimens for further mechanical characterization (the bottom figure shown here). The obtained force-displacement curves confirm the toughening effect of the nacre-like architecture, as evidenced by the strain-hardening behaviour of the material after crack initiation (panel b). The large and small specimens were compared in terms of the apparent fracture toughness $\left(K_{\mathrm{IR}}^{\mathrm{a}}\right)$, which is a well-established parameter to quantify the crack-growth resistance of brittle materials undergoing strong crack deflection and branching. Our results show that the average apparent fracture toughness for the 5-mm-thick samples is only $6 \%$ lower than the value obtained for the 3-mmthick specimens (Fig. 2c). Although further experiments are needed to test this hypothesis, our results suggest only a relatively minor effect of sample size on the fracture toughness of the nacre-like alumina fabricated by magnetically assisted slip-casting.

Continued 
Continued

a

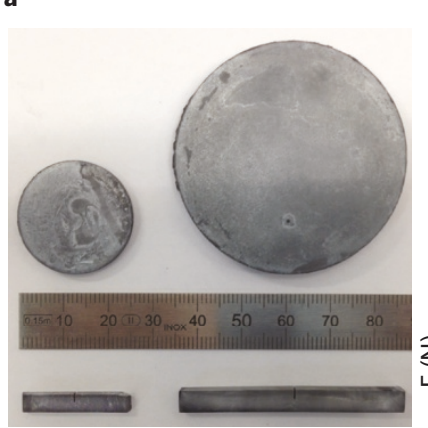

$\mathbf{f}$

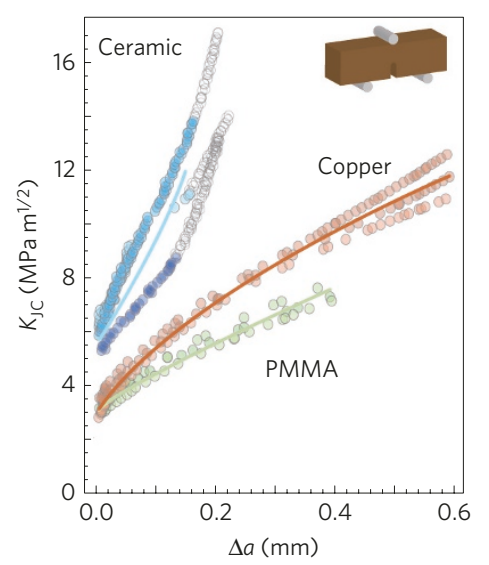

Figure 3 b

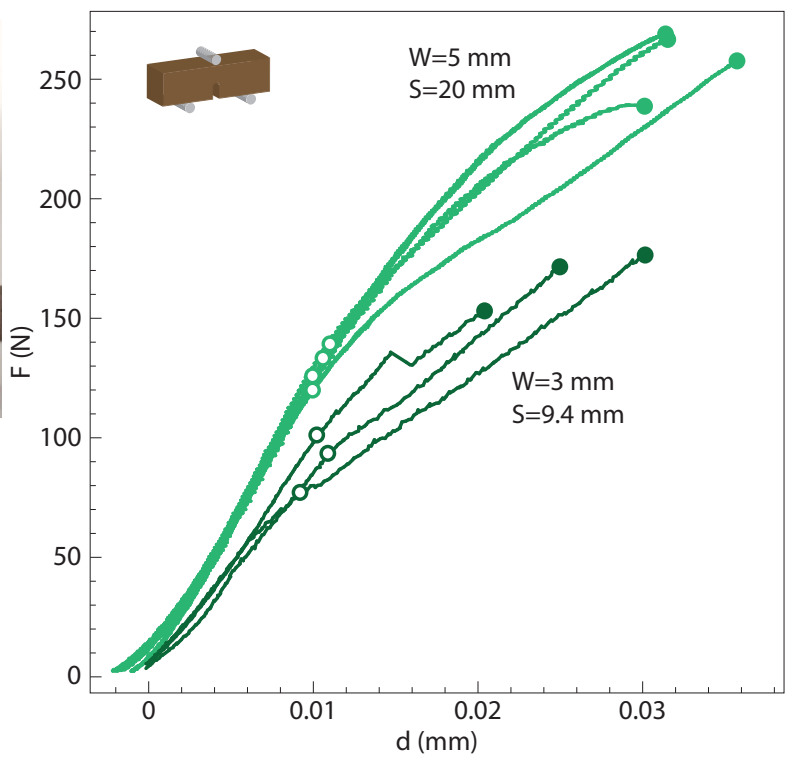

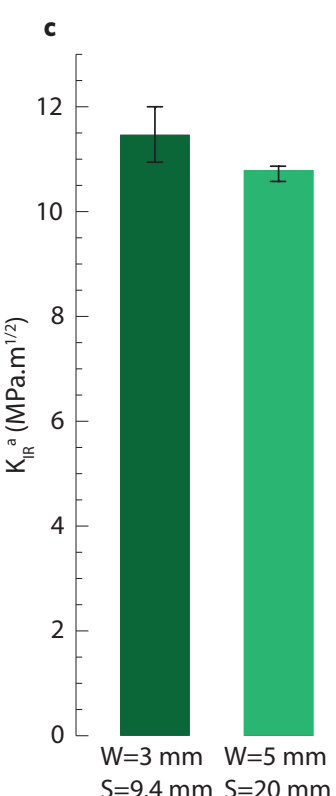

Size effects on the crack-growth resistance of nacre-like alumina. a, Samples with diameters of $20 \mathrm{~mm}$, as used in the original version of the Article, and $50 \mathrm{~mm}$, used to generate the new data reported here. b, Force-displacement curves for notched samples of two different dimensions. W, thickness; S, setup span. Empty circles indicate the first nonlinearity in the curve, which corresponds to the beginning of the fracture process for each sample. Full circles represent the end of stable crack propagation and the load used to calculate the apparent fracture toughness $\left(K_{\mathbb{R}}^{a}\right) . \mathbf{c}, K_{\mathbb{R}}^{\mathrm{a}}$ for samples of different sizes. 